\title{
ANTIBIOGRAM STUDY OF URINARY ISOLATES AMONG INPATIENTS AND OUTPATIENTS AT PUDUCHERRY STATE, SOUTH INDIA
}

\author{
Thirumurugan $\mathbf{R}^{*}$ \\ Department of Transfusion Medicine, JIPMER, Puducherry, India
}

Balu Radhakrishnan

Department of Microbiology, Indra Gandhi Government General Hospital and Post Graduate Institute, Puducherry, India

\section{Antony V. Samrot}

Department of Biomedical Sciences, Faculty of Medicine and Biomedical Sciences, MAHSA

University, Jalan SP2, Bandar Saujana Putra, 42610 Jenjarom, Selangor, Malaysia

\section{Appalaraju, V.V.S.S}

Department of Medicinal Chemistry, Faculty of Pharmacy, MAHSA University, Jalan SP2, Bandar Saujana Putra, 42610 Jenjarom, Selangor, Malaysia

\section{Raji P}

Department of Biotechnology, School of Bio and Chemical Engineering, Sathyabama Institute of Science and Technology, Jeppiaar Nagar, Chennai, Tamil Nadu, India

*Corresponding Author Email ID: thirumuruganphd@gmail.com

\begin{abstract}
The Urinary tract infections (UTI) are common in men and women and the most common in postoperative patients. In this study, antibiogram of urinary isolates among the Inpatients (IP) - 85 samples and Outpatients (OP) - 73 samples were determined. Organisms were isolated from the collected midstream urine sample and subjected for identification and evaluated for the susceptibility pattern. Mainly the Urinary Tract Infection causing organism in OP and IP was Escherichia coli. Others include Klebsiella sp., Pseudomonas sp., Proteus sp., and Staphylococcus sp in which various degrees of susceptibility patterns were recorded. Inpatients isolates showed high degree of resistance to the antibiotics used. In both IP/OP, the major uropathogen was Escherichia coli.
\end{abstract}

Keywords: Urinary Tract Infection, Antibiogram, Escherichia coli 
Antibiogram Study of Urinary Isolates Among Inpatients and Outpatients at Puducherry State, South India

Cite this Article: Thirumurugan R, Balu Radhakrishnan, Antony V. Samrot, Appalaraju, V.V.S.S, Raji P, Antibiogram Study of Urinary Isolates Among Inpatients and Outpatients at Puducherry State, South India, International Journal of Advanced Research in Engineering and Technology (IJARET), 10 (6), 2019, pp 104-111. http://iaeme.com/Home/issue/IJARET?Volume $=10 \&$ Issue $=6$

\section{INTRODUCTION}

Treatment of Urinary Tract Infection (UTI) is mainly based on the susceptibility patterns of the isolates, colony count is also important to rule out insignificant infections. The recommended significant colony count is $>10^{5} \mathrm{CFU} / \mathrm{ml}$, this is called as significant Bacteriuria [1]. The urinary tract can be infected from bacteria entering the kidneys from the bloodstream and traveling downward or from bacteria entering the urethra and traveling upward. In the Studies conducted in Ethiopia and other parts of the world showed that Escherichia coli is the major etiologic agent in causing UTI, which accounts for up to $90 \%$ of cases [2,3]. Proteus mirabilis, Klebsiella sp., Pseudomonas sp., and Gram-positive organisms are even less common in which Group B Streptococcus, Staphylococcus sp., are the recognized organisms [1]. In this study such significant growth was analyzed. Here, the characteristics of Inpatients (IP) and Outpatients (OP) urinary isolates were compared to evaluate the degree of susceptibility patterns between these two groups. Majority of the urinary tract infections in both Inpatients and Outpatients are due to Escherichia coli. Others isolates such as Klebsiella sp., Proteus sp., Pseudomonas sp., etc were also isolated among these groups (IP \&OP). Present study is aimed to find out emerging drug resistance to various routinely used antibiotics. This study was carried out at Puducherry private hospitals. There were 85 numbers of Inpatients samples and 73 numbers of Outpatients samples were analyzed. All the cases were clinically diagnosed as UTI patients.

\section{MATERIALS AND METHODS}

\subsection{Collection of Samples}

In this study, totally 158 Urine samples (85 - Inpatients samples and 73 - Outpatients samples) were collected from patients attended and hospitalized between in Private Hospitals (Clinic Nallam and Rani hospital), Puducherry. The study included that comparing the characteristics of Inpatients and Outpatients from selected urinary samples. The group comprised of 85 Inpatients and 73 Outpatients.

\subsection{Processing of Specimens and Antibiogram}

The samples were processed as described previously [4-6]. All the collected samples were streaked using a sterile inoculation loop onto MacConkey Agar and Blood Agar plates and incubated for $24 \mathrm{~h}$ at $37^{\circ} \mathrm{C}$. Grown organisms were subjected for Gram's staining, biochemical tests and identified based on Bergey's manual of systematic bacteriology. Kirby-Bauer disc diffusion method was followed to identify the drug resistance pattern [7,8]. The following discs were used (bought from HiMedia, India) - Amikacin (30mcg/disc), Ampicillin (10 mcg/disc), Cefoperazone/sulbactam ( $75 \mathrm{mcg} / \mathrm{disc})$, Ceftriaxone (30 mcg/disc), Cephotaxime (30 mcg/disc), Chloramphenicol (30 mcg/disc), Ciprofloxacin $(5 \mathrm{mcg} / \mathrm{disc})$, Gentamicin $(10 \mathrm{mcg} / \mathrm{disc})$, Nalidixic acid (30 mcg/disc), Nitrofurantoin $(300 \mathrm{mcg} / \mathrm{disc})$, Norfloxacin (10mcg/disc). 


\subsection{Measuring of Zone of Inhibition}

Zone of inhibition in mm was measured after incubation. In this study, even the organisms showed intermediate resistance were called as resistant [9].

\section{RESULTS AND DISCUSSION}

Tables 1 to 5 shows susceptibility patterns of 85 Inpatients isolates. Escherichia coli $(65 \%)$ showed high degree of sensitivity to Cefoperazone/Sulbactum and Nitrofurantoin. $46.66 \%$ of Escherichia coli strains were sensitive to Amikacin. Sensitivity to Cefriaxone, Chloramphenicol was recorded as $45 \%$. Most of the Escherichia coli strains resistant to the following antibiotics like Ampicillin (78.33\%), Nalidixic acid (76.66\%) and Ciprofloxacin (70\%). Strains of Klebsiella sp., highly susceptible to Amikacin (46.15\%) and Nitrofurantoin, Gentamicin (38.46\%). Only very few strains susceptible to Ciprofloxacin and Chloramphenicol $(30.76 \%)$. Klebsiella strains resistant to the following antibiotics such as Nalidixic acid, Ampicillin (76.92\%), Cefoperazone/Sulbactum, Chloramphenicol, Cephotaxime, Ceftriaxone, Norfloxacin (69.23\%), and Ciprofloxacin (53.84\%). Pseudomonas sp., isolated from Inpatients sample showed resistant towards many antibiotics. Nalidixic acid and Ampicillin (100\%), Cephotaxime (87.5\%), Ceftriaxone, Ciprofloxacin, Norfloxacin, Nitrofurantoin (62.5\%). This study revealed that highly effective antibiotics against Pseudomonas sp., are Cefoperazone/Sulbactum (75\%), Amikacin (50\%), Nitrofurantoin, Norfloxacin, and Ciprofloxacin (37.5\%). The genus Proteus sp., was highly sensitive to Amikacin, Nalidixic acid, Ciprofloxacin, Cefoperazone/Sulbactum. One strain was sensitive to Gentamicin. Staphylococcus sp., showed high degree of resistant to Amikacin, Ampicillin, Ciprofloxacin, Norfloxacin, Nitrofurantoin, Nalidixic acid, Chloramphenicol, Cefoperazone/Sulbactum was most effective antibiotic.

Table 1 Susceptibility Patterns of Escherichia coli in Inpatients isolates

\begin{tabular}{|c|c|c|c|c|c|c|c|}
\hline \multirow{2}{*}{ S.No } & Name of the Antibiotic & \multicolumn{3}{|c|}{ Frequency } & \multicolumn{3}{c|}{ \% } \\
\cline { 3 - 8 } & & S & I & R & S & I & R \\
\hline 1 & Amikacin(AK) & 28 & 13 & 19 & 46.66 & 21.66 & 31.66 \\
\hline 2 & Ampicillin(A) & 8 & 5 & 47 & 13.33 & 8.33 & 78.33 \\
\hline 3 & Nitrofurantoin(Nf) & 39 & 7 & 14 & 65 & 11.66 & 23.33 \\
\hline 4 & Norfloxacin(NX) & 12 & 13 & 35 & 20 & 21.66 & 58.33 \\
\hline 5 & Nalidixicacid(Na) & 4 & 10 & 46 & 6.66 & 16.66 & 76.66 \\
\hline 6 & Ceftriaxone(Ci) & 27 & 3 & 30 & 45 & 5 & 50 \\
\hline 7 & Cephotaxime(Ce) & 22 & 5 & 33 & 36.66 & 8.33 & 55 \\
\hline 8 & Gentamicin(G) & 26 & 7 & 27 & 43.33 & 11.66 & 45 \\
\hline 9 & Ciprofloxacin(Cf) & 10 & 8 & 42 & 16.66 & 13.33 & 70 \\
\hline 10 & Chloramphenicol(C) & 27 & 8 & 25 & 45 & 13.33 & 41.66 \\
\hline 11 & Cefoperazone/sulbactam(Cfs) & 39 & 15 & 6 & 65 & 25 & \\
\hline
\end{tabular}

Table 2 Susceptibility Patterns of Klebsiella sp., in Inpatients isolates

\begin{tabular}{|c|c|c|c|c|c|c|c|}
\hline \multirow{2}{*}{ S.No } & Name of the Antibiotic & \multicolumn{3}{|c|}{ Frequency } & \multicolumn{3}{c|}{ \% } \\
\cline { 3 - 8 } & & S & I & R & S & I & R \\
\hline 1 & Amikacin(AK) & 6 & 3 & 4 & 46.15 & 23.07 & 30.76 \\
\hline 2 & Ampicillin(A) & 3 & - & 10 & 23.07 & - & 76.92 \\
\hline 3 & Nitrofurantoin(Nf) & 5 & 2 & 6 & 38.46 & 15.38 & 46.15 \\
\hline 4 & Norfloxacin(NX) & 3 & 1 & 9 & 23.07 & 7.69 & 69.23 \\
\hline 5 & Nalidixicacid(Na) & 3 & - & 10 & 23.07 & - & 76.92 \\
\hline 6 & Ceftriaxone(Ci) & 3 & 1 & 9 & 23.07 & 7.69 & 69.23 \\
\hline 7 & Cephotaxime(Ce) & 1 & 3 & 9 & 7.69 & 23.07 & 69.23 \\
\hline 8 & Gentamicin(G) & 5 & 2 & 6 & 38.46 & 15.38 & 30.76 \\
\hline 9 & Ciprofloxacin(Cf) & 4 & 2 & 7 & 30.76 & 15.38 & 53.84 \\
\hline 10 & Chloramphenicol(C) & 4 & - & 9 & 30.76 & - & 69.23 \\
\hline 11 & Cefoperazone/sulbactam(Cfs) & - & 4 & 9 & - & 30.76 & 69.23 \\
\hline
\end{tabular}


Antibiogram Study of Urinary Isolates Among Inpatients and Outpatients at Puducherry State,

South India

Table 3 Susceptibility Patterns of Pseudomonas sp., in Inpatients isolates

\begin{tabular}{|c|c|c|c|c|c|c|c|}
\hline \multirow{2}{*}{ S.No } & Name of the Antibiotic & \multicolumn{3}{|c|}{ Frequency } & \multicolumn{3}{c|}{ \% } \\
\cline { 3 - 8 } & & S & I & R & S & I & R \\
\hline 1 & Amikacin(AK) & 4 & 1 & 3 & 50 & 12.5 & 37.5 \\
\hline 2 & Ampicillin(A) & - & - & 8 & - & - & 100 \\
\hline 3 & Nitrofurantoin(Nf) & 3 & - & 5 & 37.5 & - & 62.5 \\
\hline 4 & Norfloxacin(NX) & 3 & - & 5 & 37.5 & - & 62.5 \\
\hline 5 & Nalidixicacid(Na) & - & - & 8 & - & - & 100 \\
\hline 6 & Ceftriaxone(Ci) & 2 & 1 & 5 & 25 & 12.5 & 62.5 \\
\hline 7 & Cephotaxime(C) & - & 1 & 7 & - & 12.5 & 87.5 \\
\hline 8 & Gentamicin(G) & 2 & 2 & 4 & 25 & 25 & 50 \\
\hline 9 & Ciprofloxacin(Cf) & 3 & - & 5 & 37.5 & - & 62.5 \\
\hline 10 & Chloramphenicol(C) & 2 & - & 6 & 25 & - & 75 \\
\hline 11 & Cefoperazone/sulbactam(Cfs) & 6 & - & 2 & 75 & - & 25 \\
\hline
\end{tabular}

Table 4 Susceptibility Patterns of Proteus sp., in Inpatients isolates

\begin{tabular}{|c|c|c|c|c|c|c|c|}
\hline \multirow{2}{*}{ S.No } & Name of the Antibiotic & \multicolumn{3}{|c|}{ Frequency } & \multicolumn{3}{c|}{ \% } \\
\cline { 3 - 8 } & & S & I & R & S & I & R \\
\hline 1 & Amikacin(AK) & 2 & - & - & 100 & - & - \\
\hline 2 & Ampicillin(A) & - & - & 2 & - & - & 100 \\
\hline 3 & Nitrofurantoin(Nf) & - & - & 2 & - & - & 100 \\
\hline 4 & Norfloxacin(NX) & - & - & 2 & - & - & 100 \\
\hline 5 & Nalidixicacid(Na) & 2 & - & - & 100 & - & - \\
\hline 6 & Ceftriaxone(Ci) & - & - & 2 & - & - & 100 \\
\hline 7 & Cephotaxime(Ce) & - & - & 2 & - & - & 100 \\
\hline 8 & Gentamicin(G) & 1 & - & 1 & 50 & - & 50 \\
\hline 9 & Ciprofloxacin(Cf) & 2 & - & - & 100 & - & - \\
\hline 10 & Chloramphenicol(C) & - & 2 & - & - & 100 & - \\
\hline 11 & Cefoperazone/sulbactam(Cfs) & 2 & - & - & 100 & - & - \\
\hline
\end{tabular}

Table 5 Susceptibility Patterns of Staphylococcus sp., in Inpatients isolates

\begin{tabular}{|c|c|c|c|c|c|c|c|}
\hline S.No & Name of the Antibiotic & \multicolumn{3}{|c|}{ Frequency } & \multicolumn{3}{c|}{$\%$} \\
\cline { 3 - 7 } & & S & I & R & S & I & R \\
\hline 1 & Amikacin(AK) & - & - & 2 & - & - & 100 \\
\hline 2 & Ampicillin(A) & - & - & 2 & - & - & 100 \\
\hline 3 & Nitrofurantoin(Nf) & - & - & 2 & - & - & 100 \\
\hline 4 & Norfloxacin(NX) & - & - & 2 & - & - & 100 \\
\hline 5 & Nalidixicacid(Na) & - & - & 2 & - & - & 100 \\
\hline 6 & Ceftriaxone(Ci) & - & - & 2 & - & - & 100 \\
\hline 7 & Cephotaxime(Ce) & 2 & - & - & 100 & - & - \\
\hline 8 & Gentamicin(G) & - & - & 2 & - & - & 100 \\
\hline 9 & Ciprofloxacin(Cf) & - & - & 2 & - & - & 100 \\
\hline 10 & Chloramphenicol(C) & - & - & 2 & - & - & 100 \\
\hline 11 & Cefoperazone/sulbactam(Cfs) & 2 & - & - & 100 & - & - \\
\hline
\end{tabular}

Our report was similar to study done in El Batnan Medical Center, where the UTI was caused mostly by E. coli as $40.46 \%(106 / 262)$ and was most sensitive to Ciprofloxacin $(85.8 \%)$ with noticeable resistance to Ampicillin (90.6\% resistance). Klebsiella sp., was the second commonest pathogen 22.5\% (59/262) against which Ciprofloxacin and Amoxicillin were most sensitive (71.2\%) for both, with clear resistance to Ampicillin (100\%) and Cephalothin (79.7\%) [10].

Tables 6 to 10 shows susceptibility patterns of 73 Outpatients isolates. Escherichia coli $(75 \%)$ showed high degree of sensitivity to the new generation antibiotic Cefoperazone/Sulbactum. $73.33 \%$ of Escherichia coli strains were sensitive to Nitrofurantoin. 
Sensitivity to Cefriaxone was recorded as $58.33 \%$. Most of the Escherichia coli strains were resistant to the following antibiotics - Ampicillin (78.33\%), Nalidixic acid $(73.33 \%)$ and Ciprofloxacin, Norfloxacin (70\%). The strains of Klebsiella sp., were highly susceptible to Ceftriaxone (100\%) and Cefoperazone/Sulbactum, Gentamicin (71.42\%). Only very few strains were susceptible to Amikacin, Nitrofurantoin, Ciprofloxacin and Chloramphenicol (57.14\%). Like Escherichia coli, Klebsiella strains also showed high degree of resistant to the Ampicillin (85.71\%), resistance to other antibiotic such as Norfloxacin, Ciprofloxacin, Nalidixic acid which was about $42.85 \%$. The resistance pattern of Pseudomonas sp was found for following antibiotics - Chloramphenicol, Gentamicin, Norfloxacin and Ampicillin and was susceptible to Amikacin, Ciprofloxacin, Nitrofurantoin, Nalidixic acid, Cefoperazone/Sulbactum. The genus Proteus sp., was $100 \%$ sensitive to Norfloxacin, Ciprofloxacin, Gentamicin and Cefoperazone/Sulbactum. Only one Staphylococcus sp., was isolated and it was resistant to Ampicillin, Cephotaxime and sensitive to other antibiotics used in this study.

Table 6 Susceptibility Patterns of Escherichia coli in Outpatients isolates

\begin{tabular}{|c|c|c|c|c|c|c|c|}
\hline \multirow{2}{*}{ S.No } & Name of the Antibiotic & \multicolumn{3}{|c|}{ Frequency } & \multicolumn{3}{c|}{ \% } \\
\cline { 3 - 8 } & & S & I & $\mathbf{R}$ & $\mathbf{S}$ & $\mathbf{I}$ & $\mathbf{R}$ \\
\hline 1 & Amikacin(AK) & 26 & 18 & 16 & 43.33 & 30 & 26.66 \\
\hline 2 & Ampicillin(A) & 12 & 1 & 47 & 20 & 1.66 & 78.33 \\
\hline 3 & Nitrofurantoin(Nf) & 44 & 6 & 10 & 73.33 & 10 & 16.66 \\
\hline 4 & Norfloxacin(NX) & 17 & 1 & 42 & 28.33 & 1.66 & 70 \\
\hline 5 & Nalidixicacid(Na) & 13 & 3 & 44 & 21.66 & 5 & 73.33 \\
\hline 6 & Ceftriaxone(Ci) & 35 & 7 & 18 & 58.33 & 11.66 & 30 \\
\hline 7 & Cephotaxime(Ce) & 23 & 7 & 30 & 38.33 & 11.66 & 50 \\
\hline 8 & Gentamicin(G) & 28 & 3 & 29 & 46.66 & 5 & 48.33 \\
\hline 9 & Ciprofloxacin(Cf) & 15 & 3 & 42 & 25 & 5 & 70 \\
\hline 10 & Chloramphenicol(C) & 25 & 10 & 25 & 41.66 & 16.66 & 41.66 \\
\hline 11 & Cefoperazone/sulbactam(Cfs) & 45 & 10 & 5 & 75 & 16.66 & 8.33 \\
\hline
\end{tabular}

Table 7 Susceptibility Patterns of Klebsiella sp., in Outpatients isolates

\begin{tabular}{|c|c|c|c|c|c|c|c|}
\hline \multirow{2}{*}{ S.No } & Name of the Antibiotic & \multicolumn{3}{|c|}{ Frequency } & \multicolumn{3}{c|}{ \% } \\
\cline { 3 - 8 } & & S & I & R & S & I & R \\
\hline 1 & Amikacin(AK) & 4 & 1 & 2 & 57.14 & 14.28 & 28.57 \\
\hline 2 & Ampicillin(A) & - & 1 & 6 & - & 14.28 & 85.71 \\
\hline 3 & Nitrofurantoin(Nf) & 4 & 1 & 2 & 57.14 & 14.28 & 28.57 \\
\hline 4 & Norfloxacin(NX) & 4 & - & 3 & 57.14 & - & 42.85 \\
\hline 5 & Nalidixicacid(Na) & 3 & 1 & 3 & 42.85 & 14.28 & 42.85 \\
\hline 6 & Ceftriaxone(Ci) & 7 & - & - & 100 & - & - \\
\hline 7 & Cephotaxime(Ce) & 3 & 2 & 2 & 42.85 & 28.57 & 28.57 \\
\hline 8 & Gentamicin(G) & 5 & - & 2 & 71.42 & - & 28.57 \\
\hline 9 & Ciprofloxacin(Cf) & 4 & - & 3 & 57.14 & - & 42.85 \\
\hline 10 & Chloramphenicol(C) & 4 & 1 & 2 & 57.14 & 14.28 & 28.57 \\
\hline 11 & Cefoperazone/sulbactam(Cfs) & 5 & 1 & 1 & 71.42 & 14.28 & 14.28 \\
\hline
\end{tabular}


Antibiogram Study of Urinary Isolates Among Inpatients and Outpatients at Puducherry State, South India

Table 8 Susceptibility Patterns of Pseudomonas sp., in Outpatients isolates

\begin{tabular}{|c|c|c|c|c|c|c|c|}
\hline \multirow{2}{*}{ S.No } & Name of the Antibiotic & \multicolumn{2}{|c|}{ Frequency } & \multicolumn{3}{c|}{$\%$} \\
\cline { 3 - 7 } & & S & I & R & S & I & R \\
\hline 1 & Amikacin(AK) & 1 & - & - & 100 & - & - \\
\hline 2 & Ampicillin(A) & - & - & 1 & - & - & 100 \\
\hline 3 & Nitrofurantoin(Nf) & 1 & - & - & 100 & - & - \\
\hline 4 & Norfloxacin(NX) & - & - & 1 & - & - & 100 \\
\hline 5 & Nalidixicacid(Na) & 1 & - & - & 100 & - & - \\
\hline 6 & Ceftriaxone(Ci) & 1 & - & - & 100 & - & - \\
\hline 7 & Cephotaxime(Ce) & 1 & - & - & 100 & - & - \\
\hline 8 & Gentamicin(G) & - & - & 1 & - & - & 100 \\
\hline 9 & Ciprofloxacin(Cf) & 1 & - & - & 100 & - & - \\
\hline 10 & Chloramphenicol(C) & - & - & 1 & - & - & 100 \\
\hline 11 & Cefoperazone/sulbactam(Cfs) & 1 & - & - & 100 & - & - \\
\hline
\end{tabular}

Table 9 Susceptibility Patterns of Proteus sp., in Outpatients isolates

\begin{tabular}{|c|c|c|c|c|c|c|c|}
\hline \multirow{2}{*}{ S.No } & Name of the Antibiotic & \multicolumn{3}{|c|}{ Frequency } & \multicolumn{3}{c|}{$\%$} \\
\cline { 3 - 8 } & & S & I & R & S & I & R \\
\hline 1 & Amikacin(AK) & 3 & - & 1 & 75 & - & 25 \\
\hline 2 & Ampicillin(A) & 1 & - & 3 & 25 & - & 75 \\
\hline 3 & Nitrofurantoin(Nf) & 1 & - & 3 & 25 & - & 75 \\
\hline 4 & Norfloxacin(NX) & 4 & - & - & 100 & - & - \\
\hline 5 & Nalidixicacid(Na) & 2 & - & 2 & 50 & - & 50 \\
\hline 6 & Ceftriaxone(Ci) & 3 & 1 & - & 75 & 25 & - \\
\hline 7 & Cephotaxime(Ce) & 1 & - & 3 & 25 & - & 75 \\
\hline 8 & Gentamicin(G) & 4 & - & - & 100 & - & - \\
\hline 9 & Ciprofloxacin(Cf) & 4 & - & - & 100 & - & - \\
\hline 10 & Chloramphenicol(C) & 3 & - & 1 & 75 & - & 25 \\
\hline 11 & Cefoperazone/sulbactam(Cfs) & 4 & - & - & 100 & - & - \\
\hline
\end{tabular}

Table 10 Susceptibility Patterns of Staphylococcus sp., in Outpatients isolates

\begin{tabular}{|c|c|c|c|c|c|c|c|}
\hline \multirow{2}{*}{ S.No } & Name of the Antibiotic & \multicolumn{2}{|c|}{ Frequency } & \multicolumn{4}{c|}{$\%$} \\
\cline { 3 - 8 } & & S & I & R & S & I & R \\
\hline 1 & Amikacin(AK) & 1 & - & - & 100 & - & - \\
\hline 2 & Ampicillin(A) & - & - & 1 & - & - & 100 \\
\hline 3 & Nitrofurantoin(Nf) & 1 & - & - & 100 & - & - \\
\hline 4 & Norfloxacin(NX) & 1 & - & - & 100 & - & - \\
\hline 5 & Nalidixicacid(Na) & 1 & - & - & 100 & - & - \\
\hline 6 & Ceftriaxone(Ci) & 1 & - & - & 100 & - & - \\
\hline 7 & Cephotaxime(Ce) & - & - & 1 & - & - & 100 \\
\hline 8 & Gentamicin(G) & 1 & - & - & 100 & - & - \\
\hline 9 & Ciprofloxacin(Cf) & 1 & - & - & 100 & - & - \\
\hline 10 & Chloramphenicol(C) & 1 & - & - & 100 & - & - \\
\hline 11 & Cefoperazone/sulbactam(Cf) & 1 & - & - & 100 & - & - \\
\hline
\end{tabular}

Note: S-Sensitive, I-Intermediate, R-Resistance

Table 11 Frequency of Uropathogens in Outpatients and Inpatients samples

\begin{tabular}{|c|c|c|c|}
\hline \multirow{2}{*}{ S. No. } & Name of the organisms & \multicolumn{2}{|c|}{ Frequency of urinary isolates } \\
\cline { 3 - 4 } & & OP & IP \\
\hline 1 & E.coli & $60(82.19 \%)$ & $60(70.59 \%)$ \\
\hline 2 & Klebsiella sp., & $7(9.59 \%)$ & $13(15.29 \%)$ \\
\hline 3 & Pseudomonas sp., & $1(1.37 \%)$ & $8(9.41 \%)$ \\
\hline 4 & Proteus sp., & $4(5.48 \%)$ & $2(2.36 \%)$ \\
\hline 5 & Staphylococcus sp., & $1(1.37 \%)$ & $2(2.36 \%)$ \\
\hline \multicolumn{2}{|c|}{ Total } & 73 & 85 \\
\hline
\end{tabular}


Frequency of urinary isolates among Outpatients and Inpatients are presented in Table 11. There are 158 samples analyzed. Main etiological agent of UTI in Outpatients and Inpatients Escherichia coli (OP 60/730 and IP 60/73). Prevalence of organism in descending order was Escherichia coli (IP 70.59\% and OP 82.19\%), Klebsiella sp., (IP 15.29\% and OP 9.59\%) Pseudomonas sp., (IP 9.41\% and OP 1.37\%) Proteus sp., (IP 2.36\% and OP 5.48\%) and Staphylococcus sp., (IP 2.36\% and OP 1.27\%). Infection rate with Escherichia coli in these groups were almost similar, but higher number of Escherichia coli isolates observed in Outpatient samples. Infection rate by other organisms such as Klebsiella sp., Pseudomonas sp., Proteus sp., and Staphylococcus sp., were higher in Inpatients. So, these isolates may be considered as major Nosocomial pathogens.

\section{CONCLUSION}

Various bacterial isolates were isolated and identified upto genus level based on the colony morphology, Gram staining and Biochemical analysis. Results showed that bacteriuria due to Escherichia coli are more common than other organisms. Escherichia coli excreted in faeces might have entered and caused UTI. This might be due to unhygienic practices after defecation. The present study showed that Escherichia coli developed resistance to Nalidixic acid, Norfloxacin. Cefoperazone/Sulbactum and Nitrofurantoin were very effective against the most bacterial isolates. Other isolates such as Klebsiella sp., Pseudomonas sp., Proteus sp., and Staphylococcus sp., present in more numbers in Inpatients samples. The main source for these organisms may be hospital atmosphere. These isolates also isolated from Outpatients samples but with low frequency.

\section{CONFLICT OF INTEREST}

The authors have no conflict of interest.

\section{DATA AVAIALABILITY}

N/A

\section{ETHICS STATEMENT}

Since no animals or humans are used in this study, this work does not require ethical clearance.

\section{REFERENCES}

[1] K. Loh, and N. Sivalingam, Urinary tract infections in pregnancy. Malaysian Family Physician, 2, 2007, 54-59.

[2] N. Gunther, V. Lockatell, D. Jhonson, and T. Mobly, In vivo dynamics of type 1 fimbria regulation in uropathgenic Escherichia coli during experimental urinary tract infection. Infection and Immunity, 69, 2001, 2838-2846.

[3] F. Getachew, Y. Gizachew, W. Yitayih, and S. Zufan, The Prevalence and Antimicrobial Susceptibility pattern of Bacterial Uropathogens Isolated from pregnant women. European Journal of Experimental Biology, 2012, 2, 1497-1502.

[4] M. Cheesbrough, District Laboratory Practice in Tropical countries. Cambridge University Press, London. 2, 2000, 180-265.

[5] J.G. Collee, T.J. Mackie, and J.E. McCartney, Practical Medical Microbiology, 14th Ed., Kundli press, Elsevier publishers, 2012, 113-150. 
Antibiogram Study of Urinary Isolates Among Inpatients and Outpatients at Puducherry State, South India

[6] A.B. Forbes, F.D. Sahm, S.A. Weissfelt. Bailey and Scott's diagnostic Microbiology. 12th edition. Mosby publication, 2007, 14.

[7] D. Greenwood, R.C.B. Slack, and J.F. Peutherer, Medical Microbiology. 14th edition. ELBS: 1997, 781-789.

[8] M.B. Coyle, Manual of Antimicrobial Susceptibility Testing. Washington D.C. American Society for Microbiology, 2005, 25-39.

[9] U. Gonlugur, M.Z. Bakici, L. Ozdemir, I. Akkurt, S. Icagasioglu, and F. Gultekin. Retrospective analysis of antibiotic susceptibility patterns of respiratory isolates of Pseudomonas aeruginosa in a Turkish University Hospital. Annals of Clinical Microbiology and Antimicrobials, 2, 2003, 5. doi:10.1186/1476-0711-2-5.

[10] M.D. Dabobash, M.F. Attla, M. Elgarba, and A.S.A Menshawy. Antibiogram Sensitivity in Urinary Tract Infections (UTI) at El Batnan Medical Center- Tobruk - Libya. Urology and Nephrology Journal, 4(3), 2017, 00129. DOI: 10.15406/unoaj.2017.04.00129 